\title{
Homograph coding and cerebral laterality
}

\author{
KEITH A. WOLLEN, MARGARET M. COAHRAN, STEVEN D. COX, \\ and DANIEL S. SHEA \\ Washington State University, Pullman, Washington 99164
}

\begin{abstract}
Are homographs (e.g., can) more likely to be coded concretely (e.g., tin can) when presented to the right hemisphere and abstractly (e.g., I can go) when presented to the left hemisphere? To examine coding, homographs that had both concrete and abstract meanings were shown for $120 \mathrm{msec}$, after which subjects used each homograph in a phrase or sentence. The responses of male subjects were more concrete for words presented to the left hemisphere than for those presented to the right, but females showed no such laterality.
\end{abstract}

We were interested in whether homographs presented to the right hemisphere would be coded differently from the same stimuli presented to the left hemisphere. For example, will "can" be concretely coded as "tin can" when sent to one hemisphere and abstractly coded as "I can go" when sent to the other?

Rather than examine whether a single stimulus is coded differently by the two hemispheres, previous researchers have demonstrated that some types of stimuli (words) are processed more efficiently by the left hemisphere (Caplan, Holmes, \& Marshall, 1974; Orbach, 1952), whereas other types of stimuli (such as faces and line slant) are processed more efficiently by the right hemisphere (Fontenot \& Benton, 1972; Geffen, Bradshaw, \& Wallace, 1971). There has been very little research on the direct effect of concreteness on laterality. Ellis and Shepherd (1974) reported that concrete words were recognized better than abstract words when presented to the left hemisphere. Other evidence that the right hemisphere is involved with "concrete" coding comes from Day (1977, p. 526) and Seamon and Gazzaniga (1973).

In summary, various types of data show that the right hemisphere is specialized for concrete processing, whereas the left hemisphere is specialized for abstract processing. Accordingly, we expected to find codings to be more abstract when homographs were presented to the left hemisphere than when they were presented to the right hemisphere. This hypothesis was tested by presenting homographs in the left and right visual fields and then asking subjects to use each homograph in a phrase or sentence.

\section{METHOD}

\section{Subjects}

The subjects were 20 male and 20 female introductory psychology students whose participation partially fulfilled a course requirement. To qualify for participation, subjects had to

Reprint requests should be sent to Keith A. Wollen, Department of Psychology, Washington State University, Pullman, Washington 99164 meet handedness and visual criteria. Subjects were required to respond "right-handed" to 8 or more of the 10 questions on the Hannay modification of the Neurosensory Center Handedness Questionnaire developed at the University of Iowa (Hannay \& Malone, 1976). All visual tests were conducted at near point on a Bausch and Lomb Orthorater. Each subject had to have (1) $20 / 25$ visual acuity or better, (2) .5 prism diopters or less of vertical phoria in either direction, and (3) no more than 3 prism diopters of esophoria or 6 of exophoria. The 40 subjects who met the criteria were randomly assigned to four experimental groups.

\section{Materials}

The materials were 72 homographs between three and seven letters in length chosen from the norms of Wollen, Cox, Coahran, Shea, and Kirby (1980). Each homograph had at least one common abstract and at least one common concrete meaning. Each homograph was typed in capital letters with a carbon ribbon and then photographed on Kodalith Ortho film. The resulting negatives were carefully positioned in glass slide mounts. The homographs were divided into 36 pairs such that the two homographs in a pair were equated with regard to the number of letters (with only two exceptions) and were approximately equated with respect to Thorndike-Lorge (1944) frequency. In addition, words that were obvious associates (e.g., toast and jam) or that were similar in spelling (e.g., well and will) did not appear in the same pair or in adjacent pairs.

\section{Procedure}

Each subject was run individually by one of two experimenters, one male and the other female. After conducting the visual tests, the experimenter seated the subject in front of the exposure device. The exposure device consisted of an $11-\mathrm{cm}$ wide $\times 5-\mathrm{cm}$-high rear-projection screen mounted near the center of a $107 \times 122 \mathrm{~cm}$ plywood partition painted black. A $61-\mathrm{cm}$-wide $\times 122-\mathrm{cm}$-high wing was affixed to each side of the partition to occlude peripheral vision. Homographs were presented via two Kodak Carousel projectors mounted behind the screen. Exposure durations were controlled by Uniblitz 225 shutters mounted onto the front of each lens. The shutters were controlled by carefully calibrated Uniblitz 330C control units. The intensities of the projected images were reduced and equated on the left and right sides by the use of neutral density filters affixed to the front of the lens. Chin and forehead rests were situated such that a subject's eyes were approximately $40 \mathrm{~cm}$ from the screen.

Once the subject was seated at the exposure device, instructions were given describing the procedure and indicating that the purpose was to see how well people could recognize briefly flashed words. Electrodes were affixed to permit electroocculographic recording, and the subject's head was positioned in the 
headrest. Lateral movement was restricted by means of a clamp on each side of the head. Then the subject practiced fixating until able to maintain a steady fixation, which usually required 1 or $2 \mathrm{~min}$. During this practice, the experimenter provided verbal feedback concerning eye movement as revealed by the electroocculographic recording.

After fixation training, four practice trials were given. Each trial began with the onset of a 3,500- $\mathrm{Hz}$ tone and a fixation light. The fixation light consisted of a $5-\mathrm{V}$ pilot light placed behind the rear-projection screen and filtered so that it would not produce glare. To provide a specific surface upon which subjects could focus, a $1.5-\mathrm{cm}$-wide strip of black plastic tape was attached to the subject's side of the screen such that it divided the screen into a left visual field (LVF) and a right visual field (RVF). A hole that subtended a visual angle of 40 min was made in this strip, and black cross hairs (made from thin strips of tape) were placed over the hole.

The tone was turned off after $.5 \mathrm{sec}$, but the fixation light remained on. When the subject's fixation was steady, two homographs were presented simultaneously for $120 \mathrm{msec}$, one in the LVF and the other in the RVF. Each homograph was presented horizontally such that the center of the word was $3 \mathrm{deg} 10 \mathrm{~min}$ from the center of the fixation light. Accurate alignment on the screen was achieved by taping the negatives and glass. The visual angle subtended by each word ranged from $1 \mathrm{deg} 32 \mathrm{~min}$ for three-letter words to $3 \mathrm{deg} 25 \mathrm{~min}$ for sevenletter words. The fixation light was turned off $.5 \mathrm{sec}$ after the onset of the homographs.

Four seconds after the onset of the pair of homographs, the tone and fixation light were turned on again. The tone remained on for $.5 \mathrm{sec}$ and the fixation light for $1.5 \mathrm{sec}$. One second after the tone-light onset (and $5 \mathrm{sec}$ after the onset of the homographs), the recall cue was flashed for $120 \mathrm{msec}$ in either the LVF or the RVF. The subject's task was to say a phrase or sentence that used the word that was in the same visual field as the recall cue After writing the phrase or sentence, the experimenter said "other word," which was the subject's cue to use the remaining word in a phrase or sentence. The pacing of the trials was determined by the time required to record the relevant information. The subject was free to look away except when the fixation light was on. Except for the homograph exposure time, all intervals were programmed by a Tally tape reader.

After four practice trials, the experimenter asked if the subject had any questions before the main part of the experiment started. Once these questions were answered, the 32 experimental trials were given, using the same procedure as on the practice trials. The entire experiment took about $50 \mathrm{~min}$.

Each group of 20 male and 20 female subjects was divided into four subgroups to achieve appropriate balancings. Balancing insured (1) that each word appeared in the LVF for half of the subjects and in the RVF for the other half and (2) that each word was the first of the pair to be cued for recall for half of the subjects but the second one for the other half. The sequence of the homographs was random except for the elimination of obviously related words, as described earlier. The sequence of the words cued for first recall was also random, with the restriction that, for each block of eight trials, half of the first-cued words were from the LVF and half from the RVF.

\section{RESULTS AND DISCUSSION}

\section{Frequency Correct}

The mean number of correct responses was determined for each combination of sex and visual field. For this analysis, the first and second responses were combined, since there was so little difference between them. Although the second-cued response had a slightly lower frequency correct $(.13$ lower for males and .68 lower

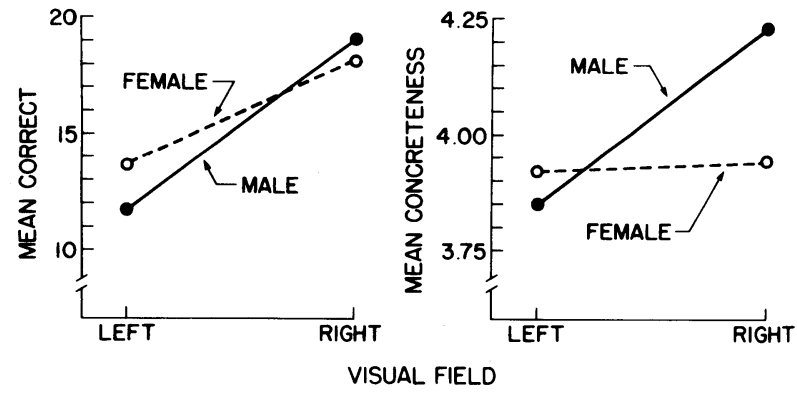

Figure 1. Frequency correct and concreteness ratings as functions of visual field and sex.

for females), the differences in number correct in LVF and RVF were virtually identical. The resulting means are shown in the left-hand portion of Figure 1.

The data were analyzed by a mixed analysis of variance that included one within-subjects factor (visual field) and two between-subjects factors, sex (male or female) and subgroup (the four subgroups used for balancing purposes). Since the subgroups were used only for balancing purposes, and since the data are only interpretable when collapsed over subgroups, subgroups were ignored in this and subsequent analyses. Although it appears that males have a slightly greater difference between LVF and RVF, the interaction of sex and visual field was not significant $[F(1,32)=3.35, p>.05]$. The effect of visual field was significant $[F(1,32)=59.50$, $\mathrm{p}<.05]$. Thus, we replicated the well-known finding of greater recall of words presented in the RVF. Finally, the effect of sex was not significant $(F<1.00)$.

\section{Concreteness Ratings}

The data of major interest are the concreteness ratings. The meaning for each correct response was assigned the concreteness rating from the norms of Wollen et al. (1980). A total of 12 of the 1,252 correct-response meanings were ambiguous and, so, were excluded from the analysis. As in the analysis of frequency correct, first-cued and second-cued responses showed similar results and were combined to gain greater stability. Incorrect responses were not included in this analysis, since errors often had only one possible meaning, thereby ruling out the possibility of showing differential coding. The means of the concreteness ratings are shown in the right-hand portion of Figure 1. The interaction between visual field and sex was significant $[\mathrm{F}(1,32)=$ $5.46, \mathrm{p}<.05]$. Consequently, the simple effects of visual field and sex were examined.

For males, concreteness ratings were significantly higher in the RVF than in the LVF $[t(32)=3.55$, $\mathrm{p}<.05$ ] . For females, however, the difference was only .02 , which was not significant $(\mathrm{t}=.19)$. Thus, males coded words more concretely in the RVF than in the LVF, whereas females showed no evidence of laterality. Analysis of the simple effects of sex showed males to have higher concreteness ratings than females in the 
RVF $[t(32)=3.62, p<.05]$, but males and females did not differ in the LVF $(\mathrm{t}=.49)$.

The finding that concreteness ratings were higher for the RVF than for the LVF was unexpected. A possible interpretation of the concreteness ratings is related to the theory (e.g., Cohen, 1973) that the left hemisphere processes information sequentially, whereas the right hemisphere processes in parallel. If so, males might tend to think of the various meanings of a homograph in serial fashion in the left hemisphere but simultaneously in the right hemisphere. If the left hemisphere does code meanings sequentially, subjects might be expected to respond with whatever meaning is dominant. It is possible to determine the mean concreteness rating of the most frequent (primary) responses and the next most frequent (secondary) responses by using the norms of Wollen et al. (1980). The resulting means of the 64 homographs (for males only) were 4.02 for primary responses and 3.08 for secondary responses. Since the primary responses tended to have high concreteness ratings, the responses to homographs shown in the RVF might be expected to be high in concreteness.

In contrast, the mean concreteness ratings for homographs shown in the LVF might be expected to be lower if several meanings were available simultaneously. In that case, the subject might decide among the several meanings on a chance basis, and abstract codings might be as likely as concrete ones. Such an outcome would produce a generally lower mean concreteness rating for homographs shown in the LVF. If this interpretation were correct, it would suggest that females would process information in parallel in both hemispheres, since the concreteness ratings were approximately equal in the LVF and the RVF.

It is, of course, possible for the left hemisphere to be better than the right hemisphere at processing abstract information and still tend to process a stimulus as concrete whenever possible. In other words, the fact that the left hemisphere is better than the right hemisphere at processing abstract information does not necessarily mean that the left hemisphere "prefers" to process in an abstract manner.

\section{REFERENCES}

Caplan, D., Holmes, J. M., \& Marshall, J. C. Word classes and hemispheric specialization. Neuropsychologia, 1974, 12, 331-337.

Cohen, G. Hemispheric differences in serial versus parallel processing. Journal of Experimental Psychology, 1973, 97, 349-356.

$\mathrm{D}_{\mathrm{AY}}$, J. Right-hemisphere language processing in normal righthanders. Journal of Experimental Psychology: Human Perception and Performance, 1977, 3, 518-528.

Ellis, H. D., \& She Phe RD, J. W. Recognition of abstract and concrete words presented in left and right visual fields. Journal of Experimental Psychology, 1974, 103, 1035-1036.

Fontenot, D. J., \& Benton, A. L. Perception of direction in the right and left visual fields. Neuropsychologia, 1972, 10, 447-452.

Geffen, G., Bradshaw, J. L., \& Wallace, G. Interhemispheric effects on reaction time to verbal and nonverbal visual stimuli. Journal of Experimental Psychology, 1971, 87, 415-422.

Hannay, H. J., \& Malone, D. R. Visual field effects and shortterm memory for verbal material. Neuropsychologia, 1976, 14, 203-209.

OrBACH, J. Retinal locus as a factor in the recognition of visually perceived words. American Journal of Psychology, 1952, 65, 555-562.

Seamon, J. G., \& Gazzaniga, M. S. Coding strategies and cerebral laterality effects. Cognitive Psychology, 1973, 5, 249-256.

ThORNDike, E. L., \& LoRge, I. The teacher's word book of 30,000 words. New York: Columbia University Press, 1944

Wollen, K. A., Cox, S. D., Coahran, M. M., Shea, D. S., \& KIRBY, R. F. Frequency of occurrence and concreteness ratings of homograph meanings. Behavior Research Methods \& Instrumentation, 1980, 12, 8-15.

(Received for publication February 17, 1980.) 